Erschienen in: Wimmer, Rainer (Hrsg.): Das 19. Jahrhundert: Sprachgeschichtliche Wurzeln des heutigen Deutsch. - Berlin, New York: de Gruyter, 1991. S. 400-427. (Institut für deutsche Sprache. WOLFGANG BRANDT Jahrbuch 1990)

\title{
Gesetzessprache. Ergebnisse einer Reihenuntersuchung
}

\section{Der Entwicklungssprung im 19. Jahrhundert}

„Die Literatur des 19. Jh. ist nicht die des 18., aber auch nicht die des 20. Jh." Mit diesem Satz, der so trivial ist, daß ihm schlechterdings nicht widersprochen werden kann, eröffnete zu meiner Studentenzeit ein Professor seine Vorlesung über die Literatur des 19. Jh. - und erntete schallendes Gelächter seines Auditoriums. Damit es mir nicht ebenso geht, möchte ich meine Eingangsthese etwas vorsichtiger und weniger banal formulieren: Die Gesetzessprache des 19. Jh. steht der des 20. Jh. wesentlich näher als der des 18 . Jh. Oder anders ausgedrückt: Die sprachlichen Veränderungen, die sich vom Ende des 19. Jh. bis zur Gegenwart vollzogen haben, sind marginal gegenüber dem Entwicklungssprung, den die Gesetzessprache im 19. Jh. gemacht hat. Unter dem Verständlichkeitskriterium betrachtet, betrifft dies sowohl im positiven Sinne die Ordnung der Textstruktur, die Verkürzung der Sătze sowie die Vereinfachung ihres Baus als auch im negativen Sinne die Herausbildung des oft beklagten übersteigerten Nominalstils mit seiner Häufung von Substantiven und dabei vor allem von abstrakten Nominalattributen.

Konkretisieren wir diesen Entwicklungssprung, der ja bei einer Textsorte erstaunt, die sich aus mancherlei Gründen sprachlichen Neuerungen gegenüber sperrig zeigt, konkretisieren wir diesen Entwicklungssprung an zwei Gesetzen, das eine vom Anfang, das andere vom Ende des 19. Jh. Hierbei handelt es sich um die kurhessische Verordnung wegen Verbesserung des Gesindewesens aus dem Jahre 1801 und um das 1900 in Kraft getretene Handelsgesetzbuch aus dem Jahre 1897. Beide Texte bilden den zeitlichen Anfangs- bzw. Schlußpunkt der Gesetze aus dem 19. Jh., die wir im Rahmen eines empirischen Projekts, das die letzten drei Jahrhunderte umfaßt, untersucht haben. ${ }^{1}$ Von den 20 Gesetzen des 20. Jh. entfallen je zehn auf die erste und die zweite JahrhundertHälfte. Kürzere und mittellange Gesetze, so die Gesinde-Verordnung, sind dabei vollständig, bei den sehr umfangreichen Gesetzen, so z.B. dem Handelsgesetzbuch, sind Auszüge von mindestens 13.000 Wörtern untersucht worden. Das Teilkorpus des 19. Jh. umfaßt insgesamt eine Textmenge von knapp 135.000 Wörtern. Bei der Auswahl spielten neben der zeitlichen Streuung nach Möglichkeit auch Inhalt und Herkunft eine Rolle. So steht das preußische Deichwesen-Gesetz (1848) neben dem

1 Die Materialbasis für das 18. Jh., sind 20, für das 20. Jh. 38 Gesetzestexte. 
Badischen Landrecht (1809), das sächsische BranntweinsteuerGesetz (1833) neben der Gewerbeordnung des Norddeutschen Bundes (1869), die hessische Blattern-Verordnung (1828) neben dem Strafgesetzbuch des Deutschen Reiches (1871). ${ }^{2}$

Kehren wir zurück zu dem ältesten und dem jüngsten Text. Die Gesinde-Verordnung verweist sprachlich weit eher ins 18 . als ins 19. Jh., ja wir können sie stellvertretend für die Gesetzessprache des 18. Jh. nehmen. Neben Besonderheiten der Rechtschreibung und Zeichensetzung (z.B. Bedürfniße vs. Zeugnisse, Feyer vs. Seite, Nahme vs. Waare, Noth vs. Gut, Livree vs. Livre; die Semikolon-Verwendung) erscheint uns zunächst die äußere Gliederung befremdlich. Denn zwei sich überschneidende Strukturen sind dem Text übergestülpt: zum einen eine Vierfach-Einteilung, gekennzeichnet mit römischen Ziffern, zum anderen eine durchgehende Paragraphen-Gliederung. Nicht selten stehen dabei die Gliederungsziffern nicht außerhalb, sondern innerhalb von Sätzen, z.B.

(1) Was sodann

II. das Vermiethen selbst betrifft; So soll Jedermann, welcher sich als Diener, Knecht ader Magd vermiethen will, [...]

Vorzüglich aber soll

§. 19.

das Gesinde in Ansehung des Feuers und Lichts sehr vorsichtig und sorgfältig seyn, $|\ldots|$

Demgegenüber ist das Handelsgesetzbuch ein systematisch durchstrukturiertes Gesetz, eingeteilt in drei Bücher zu je fünf bis acht Abschnitten, diese - wenn sachlich notwendig - noch in Titel untergliedert. Alle diese Struktureinheiten sind im Text und im Inhaltsverzeichnis mit Überschriften versehen. Die durchgezählten Paragraphen sind überschaubar, enthalten im Schnitt nur zwei bis drei Sätze. Von seiner Textstruktur und äußeren Gliederung her unterscheidet es sich in nichts von einem gut gemachten Gesetz aus unseren Tagen.

2 Außer den genannten Gesetzen bilden folgende Texte das "Teilkorpus 19. Jh.": Gesinde-Ordnung (Preußen 1810), Edilt, betreffend die bürgerlichen Verhältnisse der Juden (Preuben 1812), Mandat, den Verlauf von Arzneiwaren betrefiend (Sachsen 1823), Gesetz über die Bestrafung der Übertretungen der Ein- und Ausfubrverbote (Kurbessen 1831), Gesetz über die Verpflichtung zur Armenpflege (Preuten 1842), Gesetz über die Verhâltnisse der Miteigenthümer eines Bergwerks (Preußen 1851), Geschäfts-Ordnung für die Landstände (Kurhessen 1852), Bürgerliches Gesetzbuch (Sachsen 1863), Allgemeines Deutsches Handelngesetzbuch (Dt. Bund 1869), Verordnung zur Verhütung des ZusammenstoBes der Schifie auf See (Dt. Reich 1880), Bürgerliches Gesetzbuch (Dt. Reich 1896). 
Auch im syntaktischen Bereich sind beide Texte hervorragend geeignet, den Entwicklungssprung, von dem ich eingangs sprach, zu veranschaulichen. 1801 haben wir noch Sätze mit durchschnittlich 66 Wörtern, also mit einem Satzlängen-Wert, der fast dem der zweiten Hälfte des 18. Jh. entspricht. 1897 treffen wir dagegen auf Sätze mit nur noch 27 Wörtern im Schnitt, also auf einen Wert, der dem Durchschnitt des 20. Jh. sehr nahe kommt. Was dieser Satalängen-Unterschied konkret bedeutet, möchte ich an einer kleinen Graphik (siehe Skizze 1, S. 403) illustrieren.

Die beiden Gesamtquadrate repräsentieren die jeweilige gesamte Textmenge beider Gesetze, also die Zahl aller Wörter. Die Quadrate mit den durchgezogenen Linien in der unteren linken Ecke geben den Textanteil wieder, der in Sätzen bis zu 22 Wörtern, also in kurzen und mittellangen Sätzen, ${ }^{3}$ dargeboten wird, die Restfläche dementsprechend den jeweiligen Textanteil, der in Sätzen mit 23 und mehr Wörtern steht. Das Quadrat rechts oben greift daraus die Textmenge heraus, die in "Bandwurmsätzen" angeordnet ist, also in überlangen Sätzen mit 100 und mehr Wörtern. Der Unterschied zwischen beiden Texten ist evident. Gegenüber 1801 hat sich 1897 der Textanteil, der in kurzen und mittellangen Sätzen steht, mehr als verachtfacht. Dagegen beträgt der in superlangen Sätzen untergebrachte Anteil 1897 nur noch ein Zehntel des Anteils von 1801. Wie stark die Satzlänge mit der Satzstruktur zusammenhängt, können die gestrichelten Quadrate links unten veranschaulichen: Sie bilden den Textanteil ab, der in Einfachsätzen und Satzreihen angeordnet ist. Gegenüber der Gesinde-Verordnung ist er im Handelsgesetzbuch um das Dreizehnfache gestiegen. Dennoch steht fast drei Viertel der gesamten Textmenge auch 1897 noch in Satzgefügen auch dies charakteristisch für die Gesetzessprache des 19. Jh. Doch die Satzgefüge des Gesetzes von 1897 sind wesentlich einfacher gebaut als die der Verordnung von 1801. Insgesamt hebt sich im Vergleich beider Texte die Satztiefe von 1,76 auf 0,80. Bei der funktionalen Betrachtung der Nebensätze fallt besonders die erhebliche Zunahme der Konditionalund Restriktivsätze (von 18,5\% auf $43,6 \%$ ) und der starke Rückgang der anderen Adverbialsätze, u.a. der Kausal-, Temporal-, Konzessiv- und Modalsätze, auf (von 24,3\% auf 6,5\%).

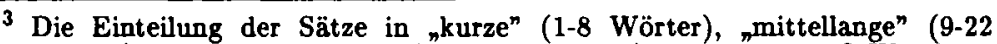
Wörter) und "lange" Sätze (über 22 Wörter) stammt von 0 .W. Hase-

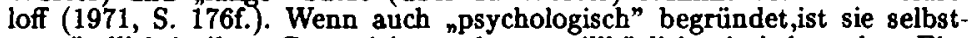
verständlich in ihrer Grenzziehung ebenso willkürlich wie jede andere Einteilung. Nicht anders steht es übrigens auch mit zeitlichen Grenzziehungen, z.B. der Einteilung nach Jahrhunderten, Jahrhunderthälften und Jahrzehnten. 
Skizze 1: Textmengen-Anteile an Satzlängen und -strukturen

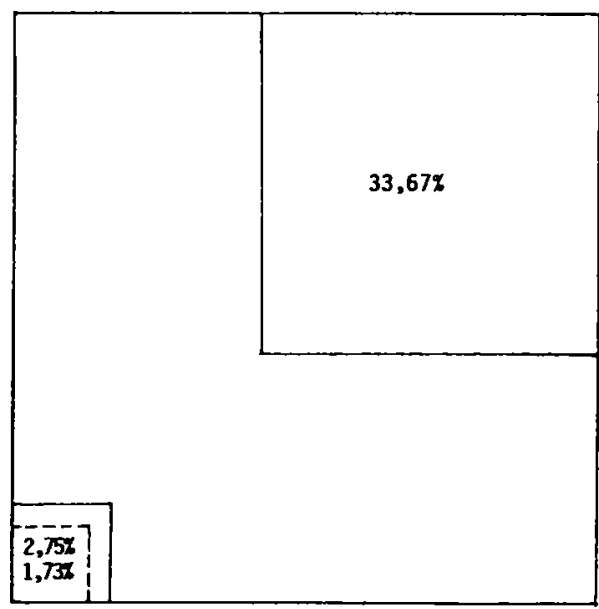

Kurhessische GesindeVerordnung 1801

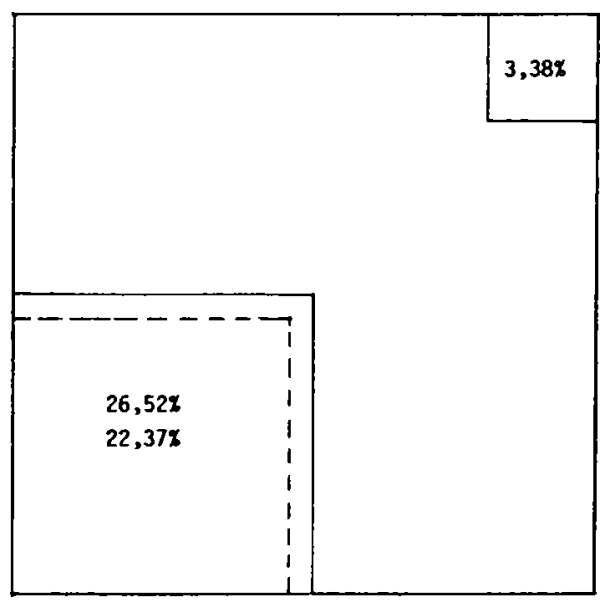

Handelsgesetzbuch 1897

Innenquadrat rechts oben: Textmenge der Sätze mit 100 und mehr Wörtern Innenquadrat links unten: Textmenge der Sätze mit bis zu 22 Wörtern Gestricheltes Innenquadrat: Textmenge der Einfachsätze und Satzreihen 
Auf der anderen Seite dokumentieren beide Gesetzestexte die forcierte Ausprägung des Nominalstils im 19. Jh. Der Anteil der Substantive an allen Textwörtern beträgt $180121,4 \%$, dagegen $189729,3 \%$. War dabei 1801 erst jedes zwölfte Substantiv des fortlaufenden Textes ein ungAbstraktum, so 1897 bereits jedes fünfte. Unter den ung-Lexemen machten dabei 1801 die Komposita nur 6,1\% aus, ihre Textfrequenz lediglich 3,5\%. Demgegenüber hat sich 1897 der Anteil der Komposita verdreifacht $(18,5 \%)$, ihre Frequenz sogar vervierfacht $(13 \%)$. Darunter sind nun auch dreigliedrige Komposita, z.B. Civilprozeßordnung und $Z$ wangsvollstreckung, die in der Gesinde-Verordnung noch nicht vorkommen. Fast verdoppelt hat sich im Handelsgesetzbuch die Zahl der Substantive, die in Attributstellung stehen $(29,5 \%$ vs. $15,5 \%$ aller Substantive). Kommen in der Gesinde-Verordnung nur maximal dreistellige Nominalattribut-Ketten vor, so im Handelsgesetzbuch Ketten bis zu sechs Gliedern.

Im Vergleich zu 1801 sind 1897 die Wörter im Schnitt länger geworden (1,94 vs. 2,10 Silben pro Wort). Jedes sechste Textwort enthält eine Silbe mehr als in der Gesinde-Verordnung. Während diese Zunahme der durchschnittlichen Wortlänge, die nicht zuletzt auf den vermehrten $\mathrm{Ge}$ brauch von Substantivkomposita zurückzuführen ist, für die Gesamtentwicklung im 19. Jh. typisch ist, gilt dies nicht generell für den Fremdwortanteil in beiden Vergleichstexten. Denn das Handelsgesetzbuch zählt zu den Gesetzen, die zwar die Übernahme deutscher Rechtsbegriffe mitgemacht haben, die aber gleichwohl von den Sprachpuristen der Jahrhundertwende wegen der Verwendung von Fremdwörtern aus dem Bereich der Gesetzesmaterie, hier also dem Handels- und Bankwesen, kritisiert wurden. ${ }^{4}$ Beispiele hierfür sind etwa: Aktie, Bilanz, Firma, Inventar, Konkurs, Prinzipal, Prokurist, Provision oder hybride Bildungen wie Handelsregister, Handlungsagent und Kommanditgesellschaft. Lateinisches Wortgut, das uns noch ganz vereinzelt in der GesindeVerordnung begegnet, z.B. officia fisci, fehlt selbstverständlich in dem Gesetz von 1897.

\section{Pragmatische Faktoren}

Dieser enorme Wandel in der Gesetzessprache des 19. Jh. ist selbstverständlich bedingt durch außersprachliche, pragmatische Entwicklungen. Besondere Auswirkungen auf die Gesetzessprache haben dabei im 19. Jh. folgende, eng miteinander verzahnte Faktoren gehabt:

${ }^{4}$ Siehe z.B. L. Günther 1898, S. $27 \mathrm{ff}$. 
2.1. Der Übergang von der Kasuistik zur generalisierenden Normativik als Folge des rechtssystematischen Denkens

In der kurhessischen Gesinde-Verordnung von 1801 steht z.B. folgende Passage, bei der es um die Pflichten des Gesindes geht:

(3) Da aber

§. 13.

die Treue insbesondere eine der vornehmsten von diesen Pflichten des Gesindes gegen die Brodherrschaft ist; So verondnen Wir, daß alle Hausbedienten, ohne Unterschied, sie mögen bey hohen oder niedrigen Herrschaften in Kost und Lohn stehen, im Hause die Kost geniessen, oder Geld dafür bekommen und sich selbst dafür verköstigen, in ihres Herrn Hause oder ausser demselben wohnen, und Ladendiener, Gesel. len, Lehrjungen, Bedienten, Knechte oder Mägde seyn, sich nicht die geringste Veruntreuungen und Verletzungen jener Pflicht zu Schulden kommen lassen sollen. Untreue Hausbedienten aber, welche ihrer Herrschaft Geld, EBwaaren oder Feldfrüchte oder sonst etwas, wenn es auch noch so gering wäre, aus den Häusern, Scheunen, Gärten oder vom Felde entwenden, Knechte und Mägde, die den Pferden oder anderm Vieh das Futter entziehen, den zum Aussäen ohne Erlaubniß der Herrschaft das Land ackern, den Ackerlohn für sich behalten, oder auf ingend eine andere Art ihre Herrschaft betrügen, wenden [...] bestraft.

Die Verfasser bemühen sich, alle nur denkbaren Fälle und Fallgruppen aufzuführen, gleich ob es sich um Personen, Sachen oder HandIungen dreht. Die preufische Gesinde-Ordnung, obgleich nur neun Jahre später entstanden, ein vergleichsweise überaus „modernes” Gesetz, verzichtet auf die Aufzählung der einzelnen Gesinde-Arten, der Unterbringungs-, Verköstigungs- und Veruntreuungsfalle. Seine Verfasser belassen es bei den Oberbegriffen für die beiden Vertragspartner Gesinde und Herrschaft, ${ }^{5}$ tilgen jede Einzelfallbeschreibung, sprechen dafür abstrakt von Schaden. Selbstverständlich entfallt auch das Herausstellen der persönlichen fürstlichen Willenskundgebung außerhalb der Präambel. Heraus kommen zwei knappe Paragraphen:

(4) §64 Das Gesinde ist schuldig, seine Dienste treu, fleißig und aufmerksam zu verrichten.

$\S 65$ Fügt es der Herrschaft vorsätzlich, oder aus grobem oder mäßigem Versehen Schaden zu: so muß es denselben ersetzen.

Insgesamt handelt es sich um einen Systematisierungs- und Abstraktionsprozeß.

5 Man beachte: in der Passage aus der Gesinde-Verordnung - Textbeispiel (3) - variieren die Autoren Herrschaft noch durch Brodherrschaft, hohe und niedrige Herrschaft und Herr. 


\subsection{Das Entstehen großer Kodifikationen}

Hierbei sind zwei unterschiedliche Gründe bzw. Notwendigkeiten zu unterscheiden. Auf der einen Seite handelt es sich um die Zusammenfassung und die Reform des Rechts im materiellen und im juristischen Bereich. Dieser ProzeB setzte im 18. Jh. ein, bot mit dem preufischen Allgemeinen Landrecht von 1794 aber erst das große Vorbild für das 19. Jh. und führte z.B. im Zivilrecht zum Badischen Landrecht von 1809, dem Bürgerlichen Gesetzbuch des Königreichs Sachsen von 1863-65 und schließlich zum BGB von 1896. Die Gründe waren rechtsphilosophischer, reformatorischer und rein praktischer Natur: so "das Systemstreben des Vernunftrechts und das zunehmende Unbehagen an einer sich geheimwissenschaftlich gebenden Rechtspflege" (Hattenhauer 1987, S. 34), der Wunsch, Gerichte und Verwaltung durch bessere Rechtskenntnis der Bürger zu entlasten, die Forderung nach allgemeiner Rechtssicherheit und die Notwendigkeit materieller Reformen.

Der praktische Nutzen der Kodifikationen für Gesetzesbefolger wie Gesetzesanwender ist offensichtlich: Bevor z.B. in Sachsen 1863 im Rahmen des Bürgerlichen Gesetzbuches in 167 Paragraphen das Scheidungsrecht systematisch und generell geregelt wurde, mußte man sich aus dem Codex Saxonicus die Einzelregelungen zusammensuchen, die häufig in Form von Rescripten ergangen waren, und notfalls ein neues Rescript des Kammergerichts erbitten.

Auf der anderen Seite erzwangen die Veränderungen im staatlichen und wirtschaftlichen Bereich, so vor allem die Ausdehnung Preußens, die Entstehung des Norddeutschen Bundes und vor allem dann die Reichsgründung, eine Rechtsvereinheitlichung für den jeweiligen Geltungsraum. In diesem Zusammenhang sei an die Gewerbe-Ordnung des Norddeutschen Bundes von 1869, an das Strafgesetzbuch von 1871, das BGB und das Handelsgesetabuch erinnert.

Zusammengefaßt können wir von einem Vereinheitlichungsprozeß sprechen.

\subsection{Der Adressaten-Bezug}

Im 18. Jh. mehrten sich die Stimmen, die forderten, die Gesetze müften von den Gesetzesbefolgern, also zumindest von den gebildeten juristischen Laien, verstanden werden. Diese Forderung gipfelte nicht nur in der berühmten Kabinetts-Ordre Friedrichs des Großen von 1780, sondern wurde auch im Allgemeinen Landrecht von den Reformern Carl Gottlieb Svarez, Johann Heinrich Casimir Graf von Carmer und Ernst Ferdinand Klein mustergültig verwirklicht. Dies geschah u.a. dadurch, daß 
die Landrecht-Verfasser die Sprachkritik aufnahmen, die zuvor schon etwa Karl Ferdinand Hommel oder Josef von Sonnenfels am schwülstigen Kanzleistil geübt hatten. So macht der Leipziger Rechtsprofessor Hommel 1765 aus seinem Herzen keine Mördergrube:

Ich hasse vor allen Dingen jene ellenlangen Schachtelsätze, in denen vieles auf einmal gebracht werden soll, und jene gewundenen Ausdrücke, 'die außer einer Sibylle niemand verstehen kann'. Nichts macht einen Vortrag verworrener und unverständlicher, als wenn man in einen Satz zehn andere mengt. Vor diesem Fehler, der den Juristen eigen ist, scheint Seneca die Gesetzgeber mit folgenden Worten zu warnen: 'Man mische nicht Gesetz in Gesetz'.6

Ganz ähnlich äufert sich Sonnenfels, u.a. Inhaber eines Lehrstuhls für Geschäftsstil und „Staatsstilist” am Wiener Kaiserhof:

Ich glaube, daß zu längeren Perioden als von zwey Gliedern bey Gesetzen nur selten ohne Abbruch der Deutlichkeit Gelegenheit seyn wird. Das Volk, für welches die Gesetze geschrieben sind, versteht nicht, was es nicht auf einmal zu umfassen făhig ist.

Und an anderer Stelle warnt er vor den negativen Folgen, die nverworrene, undeutliche Gesetze auf das Wohl des gemeinen Wesens und des einzelnen Bürgers" haben, „wenn sie wegen Dunkelheyt und Zweydeutigkeit ganz nicht oder nicht auf einerley Weise befolgt werden". ${ }^{8}$

Es ist gewiß kein historischer Zufall, daß diese kritischen, Syntax und Lexik der Gesetze betreffenden Überlegungen gerade in PreuBen unter dem aufgeklärten Monarchen Friedrich auf fruchtbaren Boden fielen. Denn die Forderung nach sprachlicher und gedanklicher Knappheit, Klarheit und Einfachheit der Gesetzessprache und damit nach Verständlichkeit für die Untertanen deckte sich nicht nur mit den vernunftrechtlichen Postulaten der französischen und deutschen Aufklärung, sondern auch mit den auf Effizienz abzielenden Sprachbedürfnissen des preußischen Militär- und Verwaltungsstaates. Dennoch hatten sich die Landrecht-Verfasser nach Friedrichs Tode (1786) im inhaltlichen und sprachlichen Bereich gegen die Kritik der konservativen, am Kanzleistil hängenden Kräfte zu wehren, die in Friedrich Wilhelm II. mehr und mehr ihre Stütze fanden. Das Landrecht ließ der neue König zwar schließlich passieren, doch strich

${ }^{6}$ K.F. Hommel 1975, S. 124f. - Das Sibylle-Zitat ist nach Polleys Anmerkung auf S. 124 der Plautus-Komödie „Pseudolus” entnommen.

7 Siehe G. Kleinheyer/J. Schröder 1976, S. 250-255.

8 J. von Sonnenfels, Über den Geschäftsstil, 2. Auf. 1785; zitiert nach H. Hattenhauer 1987, S. 47. 
er im Publikationspatent die Bürger-Passagen, stufte den Titel Allgemeines Gesetzbuch zum herkömmlichen Landrecht herunter, das als Adressaten nicht wie geplant den Bürger, sondern den Juristenstand haben sollte. ${ }^{9}$

Die Adressatenfrage wurde aber weder von den preufischen Reformern noch von ihren Gegnern entschieden. Es war vielmehr „die Verwissenschaftlichung von Recht und Rechtsdenken" (Wassermann 1981, S. 133), die vor allem Carl von Savigny, der "unbestrittene geistige Mittelpunkt" der Historischen Rechtsschule (Hattenhauer/Buschmann 1967, S. 16), durch seine rechtsphilosophischen Arbeiten und sein praktisches Wirken im preuBischen Staatsrat im Deutschland des 19. Jh. durchsetzte. Hattenhauer (1987, S. 66) bringt diesen Prozeß auf die schöne Formel: „Aus der Sprachkunst des Rationalismus sollte die Kunstsprache der Rechtswissenschaft werden." Wenn auch am Ausgang des Jahrhunderts ein starkes Interesse an Sprachkultur der Hauptgrund für jahrelanges Feilen an der Sprache des BGB war und zweifellos "die soziale Schicht des Bildungsbürgertums mit seiner Lesekultur stark angewachsen war" (Stickel 1984, S. 42), so führte der im BGB verwirklichte "hohe Abstraktionsgrad seiner Rechtsbegriffe" doch dazu, daß "das BGB kein volkstümliches Gesetzbuch geworden ist" (Kaufmann 1984, S. 178). Dieser Tatsache waren sich bereits die Zeitgenossen voll bewuBt. So schreibt der Gießener Professor der Rechte Louis Günther 1898:

Denn darüber herrscht wohl unter den einsichtigen Sachkundigen zur Zeit kaum noch eine Meinungsverschiedenheit, dass die Mehrzahl unserer neuern Reichsgesetze keine dem Volke allgemein verständliche und gewohnte Sprache redet. Auch das neue bürgerliche Gesetzbuch kann von diesem Urteile leider nicht ausgenommen werden (S. 19).

Der Versuch der Naturrechtler, Volksgesetzbücher zu schaffen und damit die Forderung nach Verständlichkeit für den Bürger zu erfüllen, erweist sich als Episode. Eindeutig wird der Adressatenkreis der Gesetzesanwender in Justiz, Verwaltung und Wissenschaft zu Lasten der Gesetzesbefolger, also der Bürger, bevorzugt. Die Herausbildung des extremen Nominalstils ist die sprachliche Folge dieser Entscheidung.

Wir können folglich in dem Zielkonflikt-Dreieck "Präzision, Effizienz, Verständlichkeit"10 von einem Präzisions- und Effizienz-Prozeß sprechen, der bis heute - trotz aller andersartigen Beteuerungen unserer Politiker - weiterwirkt.

9 Zum Allgemeinen Landrecht und den Verhältnissen in Preufen siehe H. Hattenhauer 1970 und 1987, S. 47-63.

10 Siehe hierzu W. Otto 1978, S. 11ff. und 1981, S. $49 \mathrm{ff}$. 


\subsection{Der Sprachpurismus}

Eng verknüpft mit der Adressatenfrage ist der ProzeB, die Gesetzessprache von fremdem Wortgut zu reinigen. Hier setzte das 19 . Jh. nicht nur konsequent die Bestrebungen des 18. Jh. fort, nämlich die lateinischen Termini des Römischen Rechts vollständig durch deutsche Rechtsbegriffe zu ersetzen, sondern in zunehmendem Maße wurde die "Reinigung" auch auf Fremdwörter ausgedehnt. Stand zunächst diese Entwicklung eindeutig im Zeichen naturrechtlicher Postulate und praktischer Erfordernisse, diente also der Maxime "Verständlichkeit für alle", so war diese Entwicklung vor allem nach der Reichsgründung in verstärktem Maße nationalistisch motiviert. ${ }^{11}$ Die "deutsche" Sprache und folglich auch die deutsche Gesetzessprache hatten gefalligst "deutsch" zu sein. Wie sehr dieser Sprachnationalismus gegen Ende des Jahrhunderts die Gemüter beherrschte, belegen u.a. zahlreiche Vorschläge, die 1895 für den Wahlspruch des Allgemeinen Deutschen Sprachvereins eingereicht wurden, z.B.

Deutsch sei des Deutschen Rede.

Unnützem Fremdwort Fehde (F. van Hoffs)

Kein Fremdwort braucht ein deutscher Mann,

Für das, was deutsch er sagen kann. (E. Funk)

Willst $D u$ ein guter Deutscher sein,

So sprich auch Deine Sprache rein. (C. G.)

Oder 1896 richtet Richard Jahnke folgende Verse ${ }_{\text {An }}$ das deutsche Volk":

Der Gott, der Deutschland groß gemacht,

Befreit aus Welschlands Banden,

Daß hoch und hehr in neuer Pracht

Das deutsche Reich erstanden:

Der will nicht, daß ein fremdes Wort

Die deutsche Rede schände.

l...)

Drum wirf ihn weg, den fremden Tand, und schwör ihm heiße Fehde:

Dem deutschen Sinn im deutschen Land ziemt nur die deutsche Rede. ${ }^{12}$

11 Vgl. W. Brandt 1988a, S. 121ff.

12 Aus: Der deutschen Sprache Ehrenkranz. Dichterische Zeugnisse zur Geschichte der deutschen Sprache gesammelt und erläutert von Paul Pietsch. 3. vermehrte Aufl. Berlin 1922, S. 372 und $373 f$. 
Das Allgemeine Landrecht Preußens und das Badische Landrecht dokumentieren um 1800 die rezipientenbezogene, auf Verständlichkeit ausgerichtete Eindeutschungsphase, das BGB um 1900 die sprachkulturelle, dabei aber stark nationalistisch gefärbte Reinigungsphase.

Insgesamt findet im 19. Jh. ein unterschiedlich motivierter Anti-Fremdwort-ProzeB statt, der ebenfalls bis heute - wenn nun auch unter dem Deckmantel "Verständlichkeit” - weiterwirkt.

\section{Die Vereinfachung der Syntax}

Wie sieht nun die Entwicklung der von diesen Faktoren beeinflußten Gesetzessprache konkret aus? Herausgreifen möchte ich einige Ergebnisse unserer empirischen Untersuchung, nämlich die Vereinfachung des Satzbaus und die Herausbildung des abstrakten Nominalstils, also die zwei Entwicklungen, die auch für die moderne Gesetzessprache besonders charakteristisch sind.

Meine Eingangsthese, daß die Gesetzessprache des 19. Jh. der des 20. viel näher als der des 18 . Jh. steht, läßt sich zunächst einmal eindrucksvoll an der durchschnittlichen Satzlänge beweisen.

Skizze 2: Satzlänge (Wörter pro Satz)

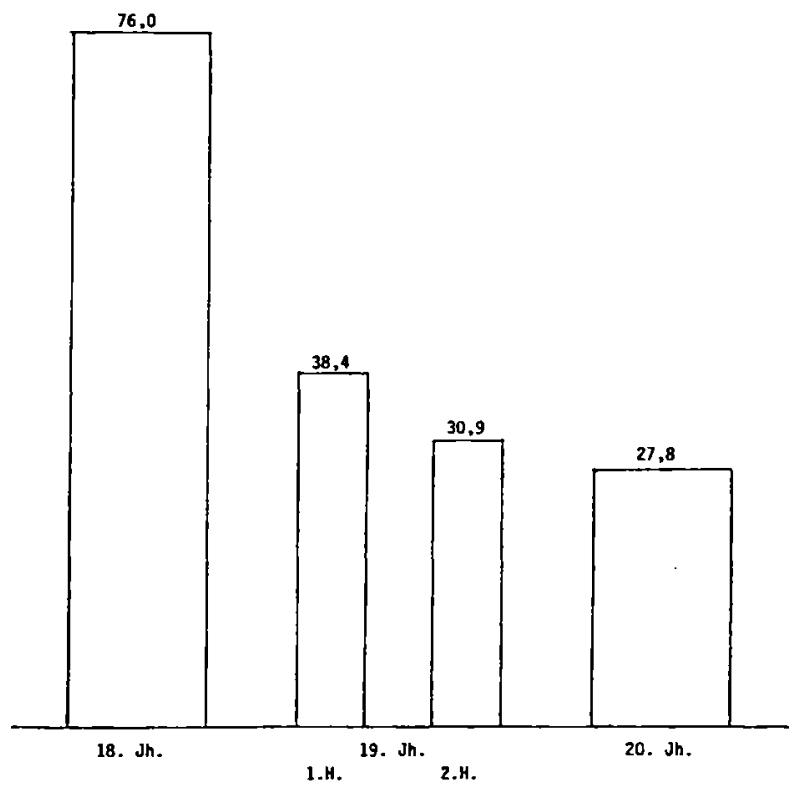


Wie Skizze 2 zeigt, vollzieht sich der entscheidende Sprung um 1800. Unter den Kriterien Stil und Verständlichkeit handelt es sich nicht nur um eine quantitative, sondern auch um eine entscheidende qualitative Veränderung. Gegenüber dieser Halbierung der Satzlänge ist die weitere Reduzierung im 20. Jh. um ganze drei Wörter in der Tat marginal; denn diese Reduzierung beträgt noch nicht einmal die Hälfte des Verkürzungsschrittes, der in der Mitte des 19. Jh. festzustellen ist.

Die entscheidende Wende stellt das Allgemeine Landrecht von 1794 dar. Es setzte zunächst zumindest für die preußische Gesetzgebung die syntaktischen Maßstäbe, die sich dann in der zweiten Jahrhunderthälfte in ganz Deutschland durchsetzen sollten. Während unter den zehn Gesetzen der zweiten Hälfte nämlich kein einziges mehr ist, dessen Satzlänge über 40 Wörter pro Satz liegt, sind es unter den zehn Gesetzen der ersten Hälfte noch vier. Nicht zufällig handelt es sich bei diesen vier ausschließlich um nichtpreußische Gesetze. Dem Vorbild des Allgemeinen Landrechts folgt außerhalb Preußens zunächst lediglich das Badische Landrecht, das allerdings als weitgehende Übersetzung des Code Napoléon dem französischen Original verpflichtet ist. Die Sätze des Badischen Landrechts und der preußischen Gesetze aus der ersten Jahrhunderthälfte sind im Schnitt um 16 Wörter kürzer als die der sächsischen und hessischen Gesetze. In Sachsen z.B. setzt der Umbruch erst in den dreißiger Jahren ein. Das Branntweinsteuer-Gesetz von 1833 hat eine Satzlänge von 30,3 Wörtern pro Satz. Das BGB des Königreichs Sachsen von 1863 erreicht dann mit 27,4 annähernd den Wert des Allgemeinen Landrechts $(23,5)$.

Wenn man die von Fucks $(1955$, S. 236) und Arens (1965, S. 19ff.) ermittelten Satzlängen-Werte für Wissenschafts- und Erzählprosa zum Vergleich heranzieht, vollzieht sich im 19. Jh. eine Angleichung dieser drei Textsorten. In der zweiten Hälfte liegt die Gesetzessprache nur noch um zwei bzw. drei Wörter pro Satz höher. Im 20. Jh. dagegen klafft die Schere wieder um durchschnittlich fünf bzw. sechs Wörter pro Satz auseinander, und zwar entgegen dem allgemeinen schriftsprachlichen Trend von den Gesetzesverfassern verschuldet.

Zurück zur Gesetzessprache des 19. Jh. Die sprachliche Revolution, die wir an dem griffigen, aber pauschalen Wert "Satzlänge" veranschaulicht haben, ist prinzipiell nur möglich, wenn zumindest eine der beiden Bedingungen erfüllt ist:

1. Es müssen Verschiebungen bei der Wahl der Satzstrukturen stattfinden. Konkret: der Anteil der Einfachsätze muß zu Lasten der 
Satzgefüge steigen; denn im Regelfalle sind in Gesetzen Einfachsätze höchstens halb so umfangreich wie Satzgefüge.

2. Die Binnenstruktur der Satzgefüge muB einfacher werden, d.h. vor allem: das einzelne Gefüge muß weniger Nebensätze enthalten, was automatisch zu einer Verkürzung führt.

Im Falle der Gesetzessprache des 19. Jh. kommen beide Bedingungen zum Tragen.

Verdeutlichen wir uns diese Zusammenhänge und Entwicklungen an unserem 3. Schaubild (S. 414). Es zeigt die prozentualen Anteile der Gefüge und der Einfachsätze, denen wir die 1 bis $2 \%$ ausmachenden Satzreihen zugeschlagen haben. Verglichen mit dem 18. Jh. hat sich der Anteil der Einfachsätze zunächst verdreifacht, dann vervierfacht. Diese Entwicklung hat sich - wie generell in der deutschen Schriftsprache - im 20. Jh. fortgesetzt. Mindestens ebenso entscheidend, ja vielleicht noch entscheidender ist aber die Halbierung der durchschnittlichen Gefüge-Länge. Die genauen Werte stehen jeweils in den Klammern unserer Skizze. Der Vergleich mit dem 20. Jh. zeigt, dab hier keine bemerkenswerte Entwicklung mehr stattgefunden hat. Mit anderen Worten: die Gefüge-Struktur der Gesetzessprache unseres Jahrhunderts ist im vorigen ausgebildet worden. Und was für den Umfang gilt, findet seine weitgehende Entsprechung in der Binnenstruktur der Satzgefüge. Thr Bau ist "einfacher" geworden. Dabei beinhaltet die Pauschalcharakterisierung "einfacher" vier unterschiedliche Prozesse:

1. Die bereits erwähnte quantitative Abnahme der Nebensätze pro Satzgefüge. Verglichen mit der Gesinde-Verordnung von 1801, gleichsam einem Gesetz des 18. Jh., verringert sich die Zahl der untergeordneten Sätze im Jahrhundertdurchschnitt auf die Hälfte.

2. Die Verringerung der Satztiefe, also der hypotaktischen Stufung. Die Satztiefe der Gefüge sinkt im Laufe des Jahrhunderts - verglichen wiederum mit der Gesinde-Verordnung - um etwa ein Drittel. Bezieht man die Zunahme der Einfachsätze mit ein, bedeutet dies für alle Textsätze fast eine Halbierung der Satztiefe.

3. Der Rückgang der Verschachtelung. In der Gesinde-Verordnung wird noch jeder fünfte Gefüge-Hauptsatz mehr als einmal durch Nebensätze unterbrochen. In der ersten Jahrhunderthälfte geschieht dies nur noch bei jedem 10. bis 11., in der zweiten Hälfte sogar nur noch bei jedem 13. Gefüge-Hauptsatz.

4. Die weitgehende Konzentrierung auf wenige funktionale Nebensatztypen. Bereits in der Gesinde-Verordnung entfält fast die 
Hälfte aller Nebensätze auf Attribut- und Konditional- bzw. Restriktivsätze. Ihr gemeinsamer Anteil erhöht sich in der ersten Hälfte auf gut zwei Drittel, in der zweiten Hălfte auf drei Viertel. Der Anteil der zahlreichen anderen Adverbialsatztypen - in der Gesinde-Verordnung noch $27 \%$ - schrumpft dagegen im Laufe des Jahrhunderts auf 10 $\%$, der der Inhaltssätze auf $15 \%$. Demgegenüber haben sich im 20. Jh. nur noch unwesentliche Verschiebungen ergeben.

Die tabellarische Auflistung der genauen Durchschnitts- und Anteilswerte bestätigt für die syntaktische Binnenstruktur und ihre funktionale Ausprägung noch einmal unsere Eingangsthese: die entscheidenden Veränderungen haben sich nicht erst im 20. Jh., sondern bereits im 19. Jh. vollzogen.

\begin{tabular}{|c|c|c|c|c|}
\hline & Gesinde- & 19. J & & 20. Jh. \\
\hline & 1801 & 1. Hälfte & 2. Hälfte & \\
\hline Nebensätze pro Gefüge & 4,1 & 2,2 & 1,8 & 1,7 \\
\hline Satztiefe der Gefüge & 1,87 & 1,38 & 1,27 & 1,22 \\
\hline Nebensätze pro Satz & 3,9 & 1,7 & 1,3 & 1 \\
\hline Satztiefe aller Sätze & 1,76 & 1,05 & 0,88 & 0,67 \\
\hline $\begin{array}{l}\text { Gefüge-Hauptsätze mit } \\
\text { mehr als } 1 \text { eingeschach- } \\
\text { telten Nebensatz }\end{array}$ & $21,4 \%$ & $9,4 \%$ & $7,8 \%$ & $2,9 \%$ \\
\hline Attributsätze & $28,4 \%$ & $43,1 \%$ & $36,5 \%$ & $36,7 \%$ \\
\hline Konditional- und & & & & \\
\hline Restriktivsätze & $18,5 \%$ & $25,9 \%$ & $38,0 \%$ & $38,1 \%$ \\
\hline andere Adverbialsätze & $27,3 \%$ & $14,7 \%$ & $10,1 \%$ & $5,9 \%$ \\
\hline Inhaltssätze & $25,8 \%$ & $16,0 \%$ & $15,4 \%$ & $18,5 \%$ \\
\hline
\end{tabular}



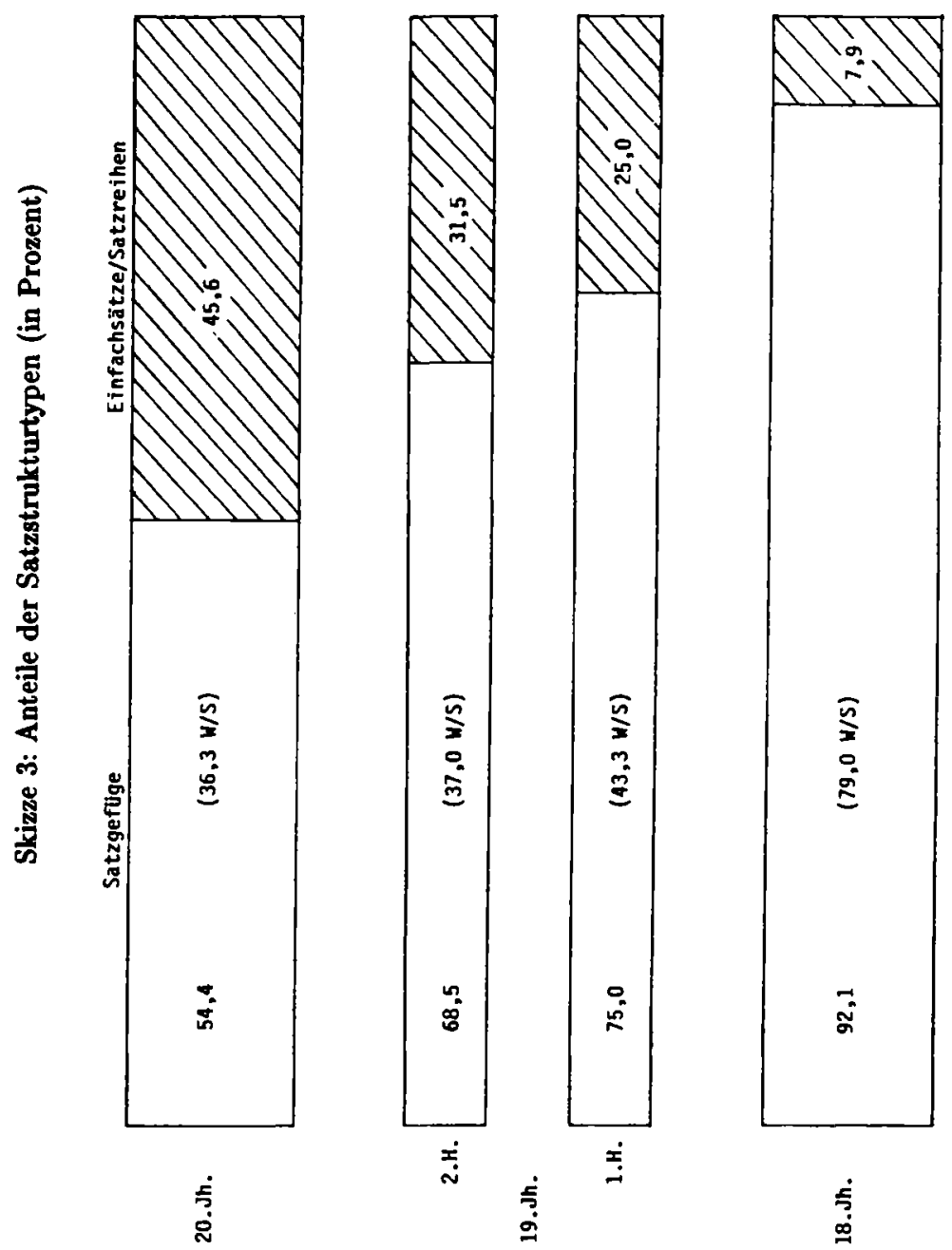


\section{Die Herausbildung des abstrakten Nominalstils}

Parallel zu diesen Entwicklungen im Satzbau verläuft im 19. Jh. die forcierte Herausbildung des Nominalstils. Wir können sie zunächst festmachen an der zunehmenden Bedeutung, die die Wortart Substantiy in der Gesetzessprache spielt. Im 18. Jh. sind 22,3\% aller Textwörter Substantive. Ihr Anteil steigt in der ersten Hälfte des 19 . Jh. auf 24,9\%, in der zweiten Hälfte auf $27,9 \%$ und erreicht im 20. Jh. 30,2 \% (siehe Skizze 6). Die höchste Steigerungsrate, nämlich drei Prozentpunkte, treffen wir innerhalb des 19. Jh. an. Der entscheidende Anstieg setzt mit den dreißiger Jahren ein und steigert sich dann in der zweiten Jahrhunderthälfte. Keines der sechs Gesetze aus den Jahren 1801 bis 1830 erreicht die $25 \%$-Marke. Dagegen liegt nur eines der 14 Gesetze aus den folgenden 70 Jahren unter diesem 25prozentigen Substantiv-Anteil, dafür haben aber sechs einen Anteil von über $28 \%$.

Als pragmatische Faktoren für diese erhebliche Zunahme sind vor allem das Streben nach Systematisierung, Präzision und Effizienz anzuführen:

1. Sachverhalte werden begrifflich gefaBt, und dies kann man am besten mit der Kategorie „Substantiv” leisten.

2. Um jegliches MiBverständnis auszuschalten, greift man zur Wiederholung des Begriffes. Man verläBt sich nicht auf die Satzsemantik und den weiteren Kotext.

3. Um Einzelsätze, z.T. auch Satzpassagen aus dem Text lösen, sie also problemlos zitieren zu können, verzichtet man mehr und mehr auf die Möglichkeit der Pronominalisierung.

Folgende kurze Textpassage, der erste Abschnitt des $\S 176$ des BGB, kann alle drei Punkte gut illustrieren:

(5) Der Vollmachtgeber kann die Vollmachtsurkunde durch eine offentliche Bekanntmachung für kraftlos erklären; die Kraftloserklärung muß nach den für die offentliche Zustellung einer Ladung geltenden Vorschriften der Civilprozeßordnung veröffentlicht werden. Mit dem Ablauf eines Monats nach der letzten Einrückung in die öffentlichen Blätter wind die Kraftloserklärung wirksam.

Nach dem Semikolon im ersten Satz hätte man fortfahren können mit: „hierbei muß er sich nach den Vorschriften der Civil-Prozebordnung richten, die für die öffentliche Zustellung einer Ladung gelten". Dies geschieht aber nicht. Statt des attributiven Relativsatzes bevorzugen die Autoren die Partizipalkonstruktion, wobei es nun zwangsläufig zu der 
stilistischen Härte doppelter Präposition-Artikel-Setzung kommt: nach den für die öffentliche Zustellung einer Ladung geltenden Vorschriften. Außerdem substantivieren sie die Verbphrase für kraftlos erklären, erfinden bzw. verwenden also den in der Allgemeinsprache unbekannten Terminus Kraftloserklärung. Der folgende Satz hat nun dasselbe Subjekt wie dieser zweite Halbsatz, nämlich das Kompositum Kraftloserklärung. Dennoch verzichten die Verfasser auf die pronominale Anknüpfung, also "Sie wird wirksam", und wiederholen den abstrakten Begriff. Man beachte bitte: Dies passiert innerhalb eines überschaubaren ParagraphenAbschnitts. Wie das Textbeispiel (4) zeigt, war es 1810 noch möglich, in einem neuen Paragraphen ausschließlich das Pronomen es - für das Gesinde im vorausgegangenen Paragraphen - zu benutzen, von dem Pronomen denselben - bezogen auf Schaden im vorangestellten Konditionalsatz - ganz zu schweigen. Am Jahrhundertende oder heute würde dieser $\S 65$ wohl lauten: „Fügt das Gesinde der Herrschait vorsätzlich oder aus grobem oder mäBigem Versehen Schaden zu, so muB das Gesinde den Schaden ersetzen." In dieser „Präzisierungs"-Fassung steigt der Substantiv-Anteil des Originals von $16,7 \%$ auf $28,6 \%$.

Unser BGB-Text bietet darüber hinaus im ersten Satz ein schönes Beispiel dafür, daß die Gesetzesautoren einerseits Personen mit ihrer jeweiligen juristischen Rolle identifizieren, hier Vollmachtgeber, andererseits den Handlungsvorgang bzw. sein Resultat dem Handlungsträger vorziehen. Indem sie das logische Subjekt ausklammern, müssen sie zwangsläufig zu einer passivischen Konstruktion greifen; hier also: die Kraftloserklärung muß veröffentlicht werden.

Nicht zufälig handelt es sich bei Kraftloserklärung um ein ungAbstraktum, und nicht zufallig sind sieben der dreizehn in unserer BGB-Passage vorkommenden Substantive Derivativa auf -ung: neben Kraftloserklärung (zweimal) noch Bekanntmachung, Zustellung, Ladung, Civilprozeßondnung und Einrückung. Denn da keine andere Wortgruppe so gut geeignet ist, den Systematisierungs- und Abstraktionsprozeb der Gesetzessprache in Sprachbegriffe umzusetzen, spiegelt die extreme Herausbildung des ung-Stils im 19. Jh. diese pragmatisch bedingte Entwicklung getreulich wider. 
Skizze 4: Anteil der ung-Substantive an allen Substantiven (in Prozent)

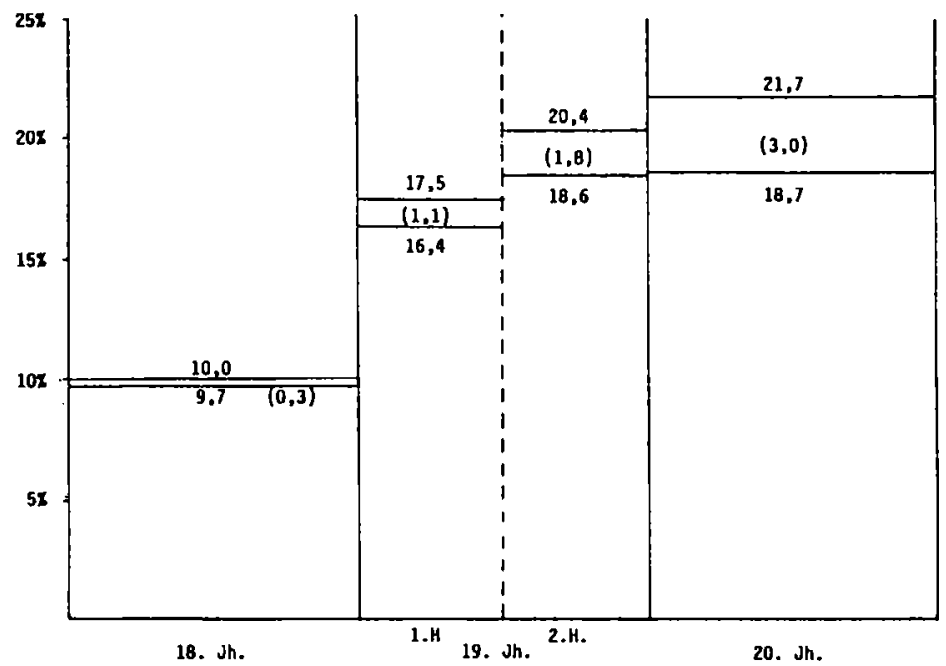

Wie die unteren Linien unserer 4. Skizze zeigen, ist bereits im 18. Jh. fast jedes zehnte Substantiv ein ung-Wort. Der entscheidende Sprung vollzieht sich wiederum in der ersten Hälfte des 19. Jh.: Jedes sechste Substantiv ist nun ein ung-Wort, in der zweiten Hälfte bereits fast jedes fünfte Substantiv. Hinzu kommt, dä im Zuge der vermehrten Komposita-Bildung im 19. Jh. die Verwendung von Komposita steigt, die als Determinans ein ung-Substantiv verwenden, also z.B. Aufkündigungsfrist, Verbietungsrecht, Vertretungsbefugnis, bisweilen auch Komposita mit ung-Determinans und ung-Determinatum, z.B. VorforderungsVerfügung, Anfechtungserklärung, Vollstreckungshandlung. Die oberen Linien unserer Graphik fassen jeweils die Frequenz aller ung-Substantive zusammen, die jeweiligen Differenzen zwischen den oberen und unteren Linien zeigen die zunehmende Tendenz, Substantiv-Komposita mit ungDeterminans zu verwenden.

Neben der vermehrten Komposita-Verwendung ist der zunehmende Gebrauch von ung-Substantiven mitverantwortlich für die Zunahme der durchschnittlichen Wortlänge, die von der ersten Hälfte des 18 . Jh. bis zur Gegenwart kontinuierlich gewachsen ist. ${ }^{13}$ Denn die ung-Wörter der Gesetze sind in ihrer übergroßen Mehrheit nicht von Simplicia, sondern

13 Siehe W. Brandt 1988b. Im 19. Jh. steigt die Wortlänge bis 1850 auf durchschnittlich 2,07 Silben pro Wort, bis 1900 auf 2,12 . 
von bereits präfigierten Verben abgeleitet. Nehmen wir das sächsische BGB von 1863 zum Beispiel. Die untersuchten 500 Sätze enthalten 159 ung-Lexeme, wobei wir zunächst die Komposita nicht berücksichtigt haben. 138 dieser Lexeme sind Präfigierungen. Unter den insgesamt 23 verschiedenen Präfixen sind ver- (30), be- (25), er- (19), an- (9) und ein(8) am häufigsten vertreten, z.B. Veräußerung, Vergehung, Verrainung oder Befriedigung, Beschädigung, Bethätigung. Hin und wieder stoßen wir auch auf Doppelpräfixe, z.B. Beurtheilung, Berücksichtigung, Übereinstimmung, Verabredung, Wiederherstellung. Dem gegenüber stehen nur 21 nichtpräfigierte ung-Lexeme, z.B. Löschung, Nöthigung, Schenkung, Weigerung. Obgleich einige von ihnen zu den zentralen Begriffen unserer analysierten Textausschnitte gehören und deswegen eine hohe Frequenz aufweisen - so Scheidung (51), Trennung (36), Handlung (26) -, haben die nichtpräfigierten ung-Substantive lediglich einen FrequenzAnteil von $23,4 \%$ an allen ung-Substantiven einschließlich der Komposita. Die im Text vorkommenden ung-Wörter sind also in ihrer überwiegenden Mehrzahl mindestens dreisilbig, zu einem Gutteil vier- und fünfsilbig, wobei bei der Pluralverwendung eine weitere Silbe hinzukommt. Beziehen wir die Komposita mit ung-Determinans oder -Determinatum mit ein, erhöht sich der Anteil der Vier- und Mehrsilber nicht unerheblich. Man nehme als Beispiele etwa zweigliedrige Komposita wie Eigentumsübertragung, Ehelichsprechung, Nichtigkeitserklärung, Scheidungserkenntnis, Veräußerungsverbot oder die 1863 noch relativ selten benutzten dreigliedrigen Komposita wie z.B. Grundstücksbeschreibung.

Der Frequenz-Anstieg der ung-Substantive dokumentiert folglich im Wortschatzbereich nicht nur den wachsenden Abstraktionsgrad der Gesetze, sondern zugleich auch die zunehmende, der begrifflichen Präzisierung dienende Differenzierung sowie die fortschreitende Informationsverdichtung.

Stellen wir zum Schluß die Frage: Mit welchem syntaktischen Mittel ordnen die Gesetzesautoren die sprunghaft angestiegene Zahl der Substantive in den Satzrahmen ein? Eine Grundmöglichkeit ist die koordinierte Aneinanderreihung, die Aufzählung, z.B. in unserer Textpassage (3) von 1801: sie mögen [...] Ladendiener, Gesellen, Lehrjungen, Bedienten, Knechte oder Mägde seyn oder aus den Häusern, Scheunen, Gärten. Derartige Aufzählungen gibt es natürlich bis heute in den Gesetzen, sei es in Form einer offenen oder einer abschließenden Enumeration, wie es in der juristischen Fachsprache heißt. Doch weit häufiger wird im 19. und 20. Jh. die zweite Grundmöglichkeit, die Subordination, benutzt, und d.h. die Verbindung von Substantiven in Form von 
Nominalattributen. ${ }^{14}$ In unserem Textbeispiel (5), der kurzen BGBPassage, kommt kein einziger Fall von Koordination vor, dafür stehen aber fünf der 13 Substantive in Attributstellung, drei - einer Ladung, der Civilprozeßordnung und eines Monats - als Genitiv-Attribute, zwei - nach der letzten Einmïckung und in die öffentlichen Blätter - als Präpositional-Attribute.

Skizze 5: Anteil der Nominalattribute an Substantiven (in Prozent)

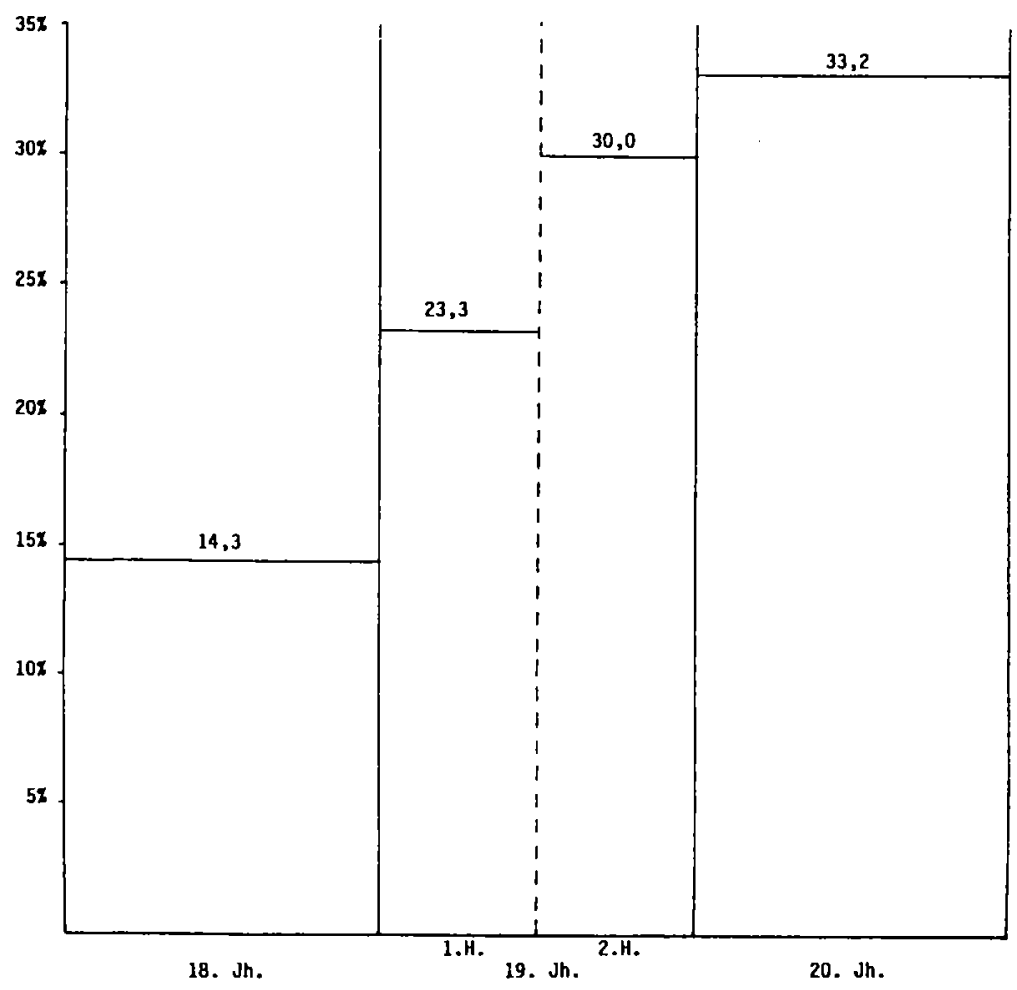

14 Auch für die Gesetzessprache gilt, was H. Sitta in der Duden-Grammatik (1984, S. 594) allgemein zum Problem der Abgrenzung von Satzglied und Attribut" schreibt: "In manchen Fällen, zumal wenn Präpositionalgefüge beteiligt sind, ist nicht immer klar zu entscheiden, ob ein Attribut oder ein selbstândiges Satzglied vorliegt." 
Wie sehr sich dieses Charakteristikum der gegenwärtigen Gesetzessprache im 19. Jh. ausgeprägt hat, zeigt unser fünftes Schaubild, das den Anteil der Substantive in Attributstellung an der Gesamtzahl aller Substantive darstellt. Wiederum ist der Anstieg im 20. Jh. relativ gering gegenüber den beiden großen Sprüngen in der ersten und zweiten Hälfte des 19. Jh. DeB in der Tat die extensive Nutzung der Subordination das syntaktische Mittel ist, um die wachsende Zahl der Substantive in den Satzrahmen zu integrieren, beweist Skizze 6.

Skizze 6: Substantiv-Anteil an Textwörtern (in Prozent)

$Z=$ in Attributstellung

$\square=$ nicht in Attributstellung

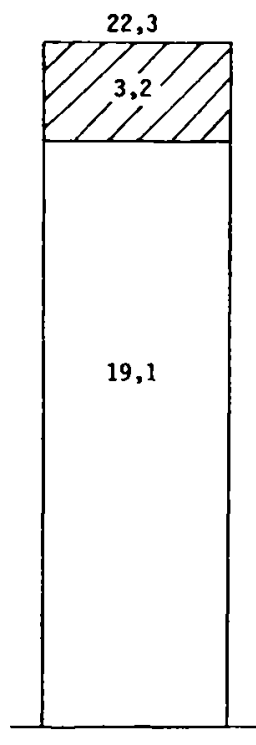

18. Jh.

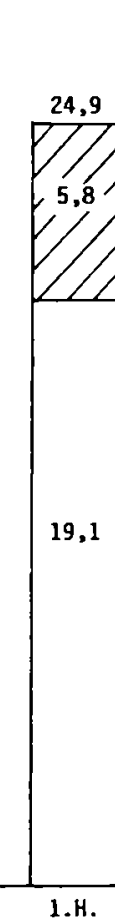

.

19. Jh.

2.H.

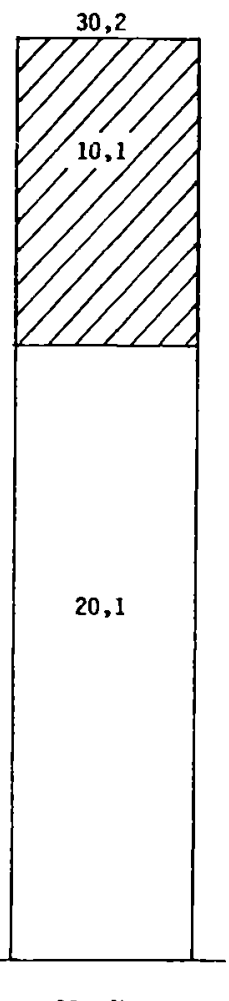

20. Jh. 
In allen Zeitabschnitten haben wir - gemessen an allen Textwörtern einen fast gleichen Substantiv-Sockel von 19 bis $20 \%$. Er stellt den Anteil der nicht-attributiven Substantive dar. Demgegenüber nimmt der schraffiert gezeichnete Anteil der Nominalattribute erheblich zu. Das bedeutet: das Mehr an Substantiven im 19. und 20. Jh. wird fast ausschlieblich in Form von Nominalattributen in den Satzrahmen eingefügt. Dieser erhebliche quantitative Anstieg der Nominalattribute ist das Produkt zweier Prozesse, die unabhängig voneinander verlaufen.

\section{Die Zunahme der Nominalattribut-Konstruktionen.}

Im 18. Jh. verteilen sich fünf Konstruktionen noch auf eine Textmenge von 200 Wörtern, im 19. Jh. auf eine von 100 Wörtern. Die Anzahl der Konstruktionen hat sich also verdoppelt.

\section{Die Zunahme der Nominalattribut-Zahl pro Konstruktion.}

Sie ist wesentlich geringer. Denn lediglich jede 13. Konstruktion enthält im 19. Jh. ein Attribut mehr als im 18. Jh.

Diese relativ geringe Steigerung verdeckt allerdings den qualitativstrukturellen Wandel. Denn im 18 . Jh. erklärt sich der vergleichsweise hohe Durchschnitt primär durch kasuistische Enumerationen in Attributstellung. Hierfür zwei Beispiele aus dem sächsischen Bäume-Mandat von 1726:

(6) eine richtige Specification von allen denen Blößen, dürren Hügeln oder sauern Grïnden, ingleichen von allen Wiesen, Feldern und andern Räumen (§ 1)

(7) Saamen von Eichen, Buchen, Ahorn, Birken, Erlen, Linden, Kiefern, Tannen, auch allerhand Obst-Körnern (§ 13)

Dagegen gewinnen im 19. Jh. hierarchisch gebaute und in sich verschachtelte Attributketten mehr und mehr an Gewicht. Die Konstruktion Ablauf eines Monats nach der letzten Einrückung in die offentlichen Blätter aus unserem Textbeispiel (5) besteht neben dem Konstruktionskopf Ablauf aus drei formal und funktional selbständigen Nominalattributen, wobei jeweils das nachfolgende dem vorausgegangenen Substantiv untergeordnet ist und es so näher spezifiziert. Die Konstruktion enthält zwar nur drei Nominalattribute, ist aber dreistellig und hat obendrein die Konstruktionstiefe „drei”. Demgegenüber bilden die sechs bzw. neun koordinierten Nominalattribute der beiden Bäume-MandatKonstruktionen jeweils nur eine Stelle auf der ersten Unterordnungsstufe.

Nun ist die Struktur nominaler Fugungsketten noch relativ leicht zu durchschauen, wenn sie dem Grundprinzip der Kettenbildung in Rein- 
form folgt, nämlich „umgekehrt angelegt" zu sein "wie ein zusammengesetztes Substantiv" (Grosse 1983, S. 20). Dies geschieht bei dem BGBBeispiel oder in folgender Konstruktion aus dem $\S 141$ des Handelsgesetzbuches:

(8) im Falle

$$
\begin{array}{ccc}
\text { der Eröffnung } & \\
& \text { des Konkurses } & \\
& \text { über das } & \\
& \text { Vermögen } & \\
& & \text { eines } \\
& & \text { Gesellschafters }
\end{array}
$$

Ausgehend von dem „Kopf" Falle steigt die Konstruktion unter Einhaltung der linearen Reihenfolge vier Hierarchisierungsstufen herab. Das aus den Gliedern dieser Konstruktion gebildete Kompositum hat die umgekehrte lineare Anordnung und steigt dementsprechend auf.

konkurs eröffnungs fall
Gesellschafter vermögens

Wesentlich schwieriger durchschaubar sind aber Nominalattribut-Konstruktionen, in denen Doppelabhängigkeiten und Verschachtelungen vorkommen. Bildlich gesprochen: Gehen wir bei der Konstruktion (8) die Treppe Stufe für Stufe herab, so müssen wir bei den komplexeren Strukturen treppab und treppauf steigen, beim Treppauf häufig springen und bisweilen auf einer Stufe Spagatschritte machen. Hierfür zwei Beispiele aus dem Handelsgesetzbuch:

(9) Besondere Bestimmungen der Satzung über die Befugniß des Vorstandes zur Vertretung der juristischen Person oder über die Zeitdauer des Unternehmens ( $\$ 33)$

(10) besondere Bestimmungen über die Zeitdauer der Gesellschaft ader über die Befugniß der Mitglieder des Vorstandes oder der Liquidatoren zur Vertretung der Gesellschaft ( $\$ 198)$

Bezeichnen wir die Ebenen der Konstruktionstiefe mit römischen Ziffern, die Stellen in der Reihenfolge der linearen Textabfolge mit arabischen Ziffern und die Glieder einer Stelle mit Kleinbuchstaben, erhalten wir für die Beispiele (9) und (10) die auf der nächsten Seite abgebildeten Strukturskizzen. 
Strukturskizzen fur die Beispiele (9) und (10)

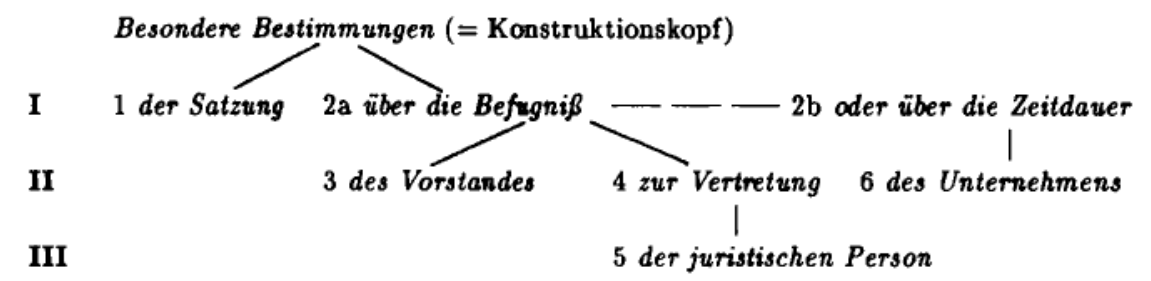

besondere Bestimmungen

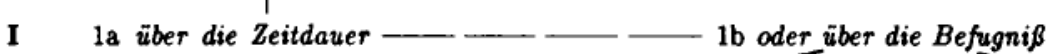

II 2 der Gesellschajt

III

3a der Mitglieder - - 3b oder der Liquidatoren 1

4 des Vorstandes
5 zur Vertretung

6 der Gesellschaft 
Beide Konstruktionen haben dieselbe Stellenzahl „sechs" und Tiefe "drei", sind aber völlig unterschiedlich gebaut. Mit zunehmender Stellenzahl steigt folglich die Vielfalt des Konstruktionsbaus.

Doch die Auflösung der Struktur stellt nur ein Verstehensproblem dar. Ebenso gewichtig ist die semantische Interpretation der einzelnen Abhängigkeitsverhältnisse. Bei den Präpositionalattributen helfen bis zu einem gewissen Grade die Präpositionen weiter, z.B. Bestimmungen über die Zeitdauer $=$ "worüber bestimmen?" oder Befugniß zur Vertretung $=$ „wozu befugt sein?". Jedoch verweist Grosse (1983, S. 18) zu Recht auf "die Verwendungsvielfalt einzelner Präpositionen" in der Gesetzessprache und fragt, "ob hier fachlich bedingte semantische Unterscheidungen festzustellen sind, ob die Variabilität des Gebrauchs unbedachter Schreibweise entspringt oder ob man aus stilistischen Erwägungen variiert". So läBt sich in unserem Beispiel (8) Konkurs über das Vermögen gewif nicht mit der Paraphrasierungsfrage "worüber Konkurs machen, worüber geschäftlich zusammenbrechen?" semantisch interpretieren. Die gröBten Probleme bereiten allerdings die Genitivattribute. Denn ihre formale Gleichheit verdeckt regelrecht ihre funktionale Vielfalt. ${ }^{15}$ So handelt es sich beispielsweise bei den drei Genitivattributen der Konstruktion (8) um drei grundverschiedene Funktionen: Fall der Eröfnnung = Genitivus explicativus bzw. definitionis, Eröffnung des Konkurses = Genitivus objectivus, Vermögen eines Gesellschafters = Genitivus possessivus.

Mehrstellige Konstruktionen stellen folglich ein eng geknüpftes Netz unterschiedlicher Subjekt-, Objekt- und Adverbial-Verhältnisse dar und führen zu einer enormen Informationsverdichtung. Die zunehmende Verwendung von Komposita in Attributstellung potenziert zusätzlich diese Verdichtung. Eine dreistellige Konstruktion wie z.B.

Unterbrechung durch Zustellung eines Zahlungsbefehls im Mahnverfahren (BGB § 213)

15 Ein schlüssiges, intersubjektiv überprüf- und nachvollziehbares Funktionsmodell der Genitivattribute gibt es nicht. Für die Zuordnung konkreter Einzelfălle sind häufig die Kategorigierungen, die sich stark an die lateinische Grammatik anlehnen - z.B. F. Blatz (1896, S. 358ff.) oder DudenGrammatik (1984, S. 598ff.) -, ebenso ein Notbehelf wie beispielsweise die Beschreibungsversuche von H. Brinkmann (1971, S. 69ff.) oder K.E. Heidolph (Grundzüge 1981, S. 301ff.). Insofern ist P. Eisenberg (1989, S. 248f.) voll zuzustimmen: "Man weiB nicht einmal genau, wie vollständig und systematisch die gefundene Liste von Attributtypen ist, und deshalb ist es auch kein Wunder, daB fast jede Grammatik ihre eigene Einteilung der Genitive hat." 
ist $\mathrm{ja}$ in Wirklichkeit eine verkürzte fünfstellige Kette ("Unterbrechung durch Zustellung eines Befehls zur Zahlung im Verfahren des Mahnens").

Entscheidend für die Herausbildung dieses "Nominalattributstils" ist wiederum das 19. Jh. Im 18. Jh. machen die einstelligen Konstruktionen, also die mit Tiefe „eins", noch über $90 \%$ aus. Vierstellige Konstruktionen kommen in unseren Texten überhaupt nicht vor. Nur in der Hälfte der Texte gibt es vereinzelt dreistellige Konstruktionen. Auch im ersten Viertel des 19. Jh. vermeiden die Gesetzesautoren noch weitgehend vierstellige Konstruktionen. Diese tauchen erst merklich um 1830 , fünfstellige dann ab den vierziger Jahren auf. Insgesamt steigt der Anteil der mehrgliedrigen Ketten von gut $8 \%$ in 18. Jh. auf knapp $15 \%$ in der ersten und auf gut $18 \%$ in der zweiten Hälfte des 19 . Jh. Diese Entwicklung hat sich im 20 . Jh. fortgesetzt, wo bereits jede vierte Konstruktion mehrstellig ist.

\section{Zusammenfassung}

Fassen wir zusammen: Die beiden skizzierten Prozesse, nämlich die Verkürzung und Vereinfachung des Satzbaus und die Herausbildung des übersteigerten Nominalstils, führen zu gegenläufigen Resultaten, wobei sie sich nur bis zu einem gewissen Grade gegenseitig bedingen.

Der eine Prozeb baut Komplexität ab, der andere dagegen auf. Der SatzProzeß findet Entsprechungen in der Ordnung und Gliederung der Textstruktur sowie in der Vereinheitlichung von Rechtschreibung und Zeichensetzung, der Nominalstil-ProzeB in der Konstruktion und Verwendung von Komposita, Prä- und Suffixbildungen und der damit verbundenen Zunahme der Wortlänge. Der eine fordert Verständlichkeit, der andere erschwert sie.

Vor der Folie der Gesetzessprache des 18. Jh. können wir zusammengefaBt von einer Komprimierung zunehmend abstrakter werdender Informationen in einem wesentlich enger und damit überschaubarer gewordenen Satzrahmen sprechen.

\section{Literatur}

Arens, Hans (1965): Verborgene Ordnung. Die Beziehungen zwischen Satzlănge und Wortlänge in der deutschen Erzäh/prosa vom Barock bis heute. Düsseldorf (Beihefte zur Zeitschrift „Wirkendes Wort” Bd. 11).

Blatz, Friedrich (1896): Neuhochdeutsche Grammatik mit Berücksichtigung der historischen Entwicklung der deutschen Sprache. 2. Bd. Satzlehre (Syntax). 3. völlig neubearbeitete Aufl. Karlsruhe. 
Brandt, Wolfgang (1988a): Lexikalische Tendenzen in der Gesetzessprache des 18.bis 20. Jahrhunderts, dargestellt am Scheidungsrecht. In: Deutscher Wortschatz. Lexikologische Studien. Ludwig Erich Schmitt zum 80. Geburtstag von seinen Marburger Schülern. Hrsg. von H.H. Munske, P. von Polenz, O. Reichmann, R. Hildebrandt. Berlin/New York, S. 119-150.

Brandt, Wolfgang (1988b): Die Wortlänge in der Gesetzessprache des 18. bis 20. Jahrhunderts. In: Sprache in Vergangenheit und Gegenwart. Beiträge aus dem Institut für Germanistische Sprachwissenschaft der PhilippsUniversität Marburg. Marburg (Marburger Studien zur Germanistik 9), S. 108-121.

Brinkmann, Hennig (1971): Die deutsche Sprache. Gestalt und Leistung. 2., neubearbeitete und erweiterte Aufl. Düsseldorf.

Duden Grammatik der deutschen Gegenwartssprache (1984): 4., völlig neu bearbeitete und erweiterte Aufl. Hrsg. und bearbeitet von Günther Drosdowski. Mannheim/Wien/Zürich.

Eisenberg, Peter (1989): Grundriß der deutschen Grammatik. 2., überarbeitete und erweiterte Aufl. Stuttgart.

Fucks, Wilhelm (1955): Unterschied des Prosastils von Dichtern und anderen Schriftstellern. Ein Beispiel mathematischer Stilanalyse. In: Sprachforum 1, S. 234-244.

Grosse, Siegfried (1983): Informationsdichte und Verständlichkeit in Gesetzesund Verwaltungstexten. In: Neuphilologische Mitteilungen 84, S. 15-24.

Grundzüge einer deutschen Grammatik (1981): Von einem Autorenkollektiv unter der Leitung von Karl Erich Heidolph, Walter Flämig und Wolfgang Motsch. Berlin.

Günther, L(ouis) (1898): Recht und Sprache. Ein Beitrag zum Thems vom Juristendeutsch. Berlin.

Haseloff, Otto Walter (1971): Über Wirkungsbedingungen politischer und werblicher Kommunikation. In: Kommunikation. Hrsg. von O.W. Haseloff. 2., unveränderte Aufl. Berlin (Forschung und Information 3), S. 151-187.

Hattenhauer, Hans/Buschmann, Arno (1967): Übersicht über die Privatrechtsgeschichte der Neuzeit. In: Textbuch zur Privatrechtsgeschichte der Neuzeit mit Übersetzungen. Von H. Hattenhauer und A. Buschmann. München, S. 1-22. 
Hattenhauer, Hans (1970): Einführung in die Geschichte des Preußischen Allgemeinen Landrechts. In: Allgemeines Landrecht für die PreuBischen Staaten von 1794. Textausgabe. Frankfurt/Berlin, S. 11-39.

Hat tenhauer, Hans (1987): Zur Geschichte der deutschen Rechts- und Gesetzessprache. Göttingen (Berichte aus den Sitzungen der Joachim JungiusGesellschaft der Wissenschaften e.V., Hamburg, Jg. 5, Heft 2).

Hommel, Karl Ferdinand (1975): Principis cura leges oder Des Fürsten höchste Sorgfalt: die Gesetze. Aus dem Lateinischen übersetzt und mit kurzen Erläuterungen begleitet von Rainer Polley. Karlsruhe (Studien und Quellen zur Geschichte des deutschen Verfassungsrechts. Reihe B: Quellen, Bd. 2).

Kaufmann, Ekkehard (1984): Deutsches Recht. Die Grundlagen. Berlin (Grundlagen der Germanistik 27).

Kleinheyer, Gerd/Schröder, Jan (Hrsg.) (1976): Deutsche Juristen aus fünf Jahrhunderten. Eine biographische Einführung in die Rechtswissenschaft. Karlsruhe/Heidelberg (UTB 578).

Otto, Walter (1978): Amtsdeutsch heute - bürgernah und praxisnah. 2., überarbeitete Aufl. Stuttgart.

Otto, Walter (1981): Die Paradoxie einer Fachsprache. In: Die Sprache des Rechts und der Verwaltung. Bearbeitet von Ingulf Radtke. Stuttgart (Der öffentliche Sprachgebrauch Bd. 2), S. 44-57.

Stickel, Gerhard (1984): Zur Kultur der Rechtssprache. In: Mitteilungen 10. Aspekte der Sprachkultur. Hrsg. vom Institut für deutsche Sprache. Mannheim, S. 29-60.

Wassermann, Rudolf (1981): Sprachliche Probleme in der Praxis von Rechtsetzung, Rechtspflege und Verwaltung. In: Die Sprache des Rechts und der Verwaltung. Bearbeitet von Ingulf Radtke. Stuttgart (Der öffentliche Sprachgebrauch Bd. 2), S. 128-142. 